\title{
Under Air Bi-Blade Vitrectomy: Perfectioning Vitreous Base Shaving
}

\author{
Emilio Pedrotti ${ }^{1}$, Erika Bonacci ${ }^{1}$, Arianna De Rossi ${ }^{1 *}$, Alessandra De Gregorio ${ }^{2}$, Chiara Chierego $^{1}$, \\ Jacopo Bonetto ${ }^{3}$, Marco Anastasi ${ }^{1}$ and Giorgio Marchini ${ }^{1}$
}

${ }^{1}$ Eye Clinic, Department of Neurosciences, Biomedicine and Movement Sciences, University of Verona (VR), Italy

${ }^{2}$ Ophthalmic Unit, San Bassiano Hospital, Bassano del Grappa (VI), Italy

${ }^{3}$ Eye Clinic, Merano Hospital, (BZ) Italy

*Corresponding author: Arianna De Rossi, Eye Clinic, Department of Neurosciences, Biomedicine and Movement Sciences, University of Verona, Verona, Italy, Pz. le L.A. Scuro 10, 37134, Verona (VR), Italy.

To Cite This Article: Arianna De R, Emilio P, Erika B, Chiara C, Marco A, et al., Air Bi-Blade Vitrectomy: Perfectioning Vitreous Base Shaving. 2020 - 9(1). AJBSR.MS.ID.001343. DOI: 10.34297/AJBSR.2020.09.001343.

Received: 眥 May 21, 2020; Published: 䟧 May 26, 2020

\section{State of the Art}

Complete vitreous removal is mandatory for successful vitreoretinal surgery performed for any indication: retinal detachment, macular hole [1], epiretinal membrane [2], and vitreous hemorrhage [3]. Accuracy in vitreous removal is indeed crucial to avoid tractional forces in the vitreous cavity that could lead to rhegmatogenous detachment or recurrence [4]. Many techniques have been developed to perform vitrectomy phases and several surgical instruments and vitreous substitutes have been introduced to optimize the vitreous removal and retina stabilization to reduce complications. One of the pivotal tools in vitreoretinal surgery is the vitrectome. This device allows effective removal of the hyaloid and vitreous base, core vitrectomy and membranes cutting. The key features of vitreous cutters are the blade design, the duty cycle, the cutting speed, and the tip-to-port distance. They were modified by technological developments in order to increase accuracy and reduce retinal traction [5]. Another important aid is given by unconventional vitreous substitutes, such as perfluorocarbonperfused [6], silicone oil [7] and air [8,9], that have been previously described. Surgeons can opt for their preferred combination according to personal experience and habits to guarantee higher precision, safety and efficacy. Particularly, vitreo-retinal surgery under air is increasing popularity among surgeons. Conventionally, during vitrectomy under fluid, the balanced saline solution (BSS)air exchange is performed after the vitreous base shaving to drain subretinal fluid and perform endolaser. Instead, vitrectomy under air technique involves an early BSS-air exchange: after the core and mid-peripheral vitrectomy (performed under BSS) and before vitreous base shaving [10]. This surgical choice provides many advantages guaranteed by physical characteristics of air, which are furtherly enhanced by the use of bi-blade cutter (Bausch \& Lomb, MI, Italy) for thorough vitreous removal [11]. Therefore, we encourage the air-infused bi-blade vitrectomy with 23-25 gauge instrumentation for its advantages over a traditional technique in terms of vitreous visualization, effective vitrectomy time, without significant increase in intraoperative and postoperative complication rate.

\section{The Advantages and Disadvantages of the Technique}

Surgeons are used to evaluate pros and cons on surgical management of complex cases, and vitreoretinal surgery is never to be underestimated. The main concern in this kind of surgery is to avoid iatrogenic retinal breaks, incomplete vitreous removal and intraoperative hypotony [12]. Many advantages of the technique can be attributed to the physical properties of air [10] and the functioning of the bi-blade cutter [13]. Appropriate visualization, especially of the peripheral retina is mandatory for any successful vitreo-retinal surgery and a complete vitreous base shaving. Many factors contribute to the sharpness and clarity of surgeon's vision: phakic or pseudophakic eye, pupil dilation and presence of any corneal or lens opacity [13]. Continuous infusion of BSS is commonly used to fill the vitreous chamber during surgery, but air has physical properties with many advantages over BSS in certain conditions. Difference in refractive index between air and BSS (1.00 versus 1.33) accounts for the wider field of visualization, which is useful, especially in narrow pupils. Another advantage is the physical impossibility of mixing air and vitreous (unlike BSS and vitreous, being both water-based structures). These 
characteristics make the air-vitreous interface more recognizable and visible compared with the BSS-vitreous interface, permitting easier and wider vitreous base visualization and allowing for an accurate and complete vitrectomy [10], without requirement for staining substances, like triamcinolone. In addition, under air, the clear visualisation of the vitreous column entering the cutter is even more recognizable thanks to the bi-blade cutter continuous aspiration. Another important advantage is the air's surface tension, which not only furtherly improves vitreous visualization, but limits the vitrectome aspiration force in between cuts during shaving maneuvers. This reduces risk of iatrogenic ruptures and permits a safer and more precise vitrectomy. Furthermore, in vitreous cavity air pushes the retina towards the sclera reducing its mobility and allowing the subretinal fluid outflow. So, vitreous base shaving under air permits much more stability of the retina if compared to the under BSS technique. The retina stability is even strengthened when the technique under air is associated to bi-blade cutter, if compared to the single blade vitrectomy.

When combined with the employment of bi-blade cutter, the advantages of vitreous base shaving under air are furtherly increased, in a synergic effect. The standard vitreous cutter design is characterized by a regular blade, which has a single port crossed by a single blade. In a duty cycle, the port changes from being completely open to completely closed, generating an inconsistent flow which is maximum when the port is open and null when the port is obstructed by the blade. The flow is then alternate and retinal traction can occur when the flow is interrupted. New generation vitreous cutters are named constant flow blade, because the dual blade design allows the port to be never completely obstructed, as a consequence, the flow is continuous and consistent. Furthermore, the cut rate is doubled and so the cutting speed. The aspiration flow is consistent due to the rapid cycle of opening and closing of the port, characterized by a cut rate up to 15.000 cuts per minute. The flow is not only consistent but also continuous and gentle, with lower risk of unbalance between forces resulting in a risky pulling of the vitreous in between aspirations [5]. So, the dual port, synergically acts with air physical properties to guarantee high retina stability which permits to work safely and closely during the shaving.

Besides, while vitrectomy under fluid is associated with iatrogenic ruptures in $7 \%$ of cases, in vitrectomy under air iatrogenic ruptures rate is $2 \%$ only [14]. It is noticeable that the combination of these two techniques provides a supplemental advantage to surgery success. Thanks to greater retinal stability and wider visualization by the surgeon, the indentation rate is significantly reduced in under air vitrectomy technique $(17,5 \%)$ [15]. This contributes to shorten surgery time, to reduce the dependance on a second operator [16] and to improve comfort for both patient and surgeon.

None surgical technique is devoid of downsides: the control of the intraocular pressure (IOP) during vitrectomy under air must be strict for the higher risk of hypotony. It is the main drawback of this technique because outflow via the vitreous cutter, at standard aspiration rates, is unimpeded. Machine setup should involve low vacuum (200 mmhg), high cut rate (higher than 5000 cuts if possible) and infusion pressure at $35 \mathrm{mmHg}$. Interruption of vacuum for brief periods is advisable to further reduce the risk of hypotony [10]. However Bausch and Lomb Stellaris Elite is integrated with a new function called Adaptive fluidics. Due to continuous balance between aspiration and infusion the IOP is stabilized throughout surgery [17]. Another important factor to be considered is the intraocular lens (IOL) fogging secondary to air condensation, which could compromise the retinal visualization when posterior capsule openings are present. This is due to the difference in temperature between air inflow and the vitreous cavity. In this case, it is useful to gently clean the posterior surface of the IOL with a silicone tipped cannula or with BSS through a blunt cannula. Another suggested strategy is to use an irrigating contact lens as a way of heating the anterior segment by emission of warmed irrigation [18]. Anyway, poly methyl methacrylate (PMMA) and hydrophobic acrylic IOLs are less prone to condensation compared to hydrophilic and silicone IOLs, especially after the first wipe of condensation [19]. However a steep learning curve is needed to achieve a proper visualization in pseudophakic eyes, especially if there are posterior capsule opacities or IOL edges occupying the surgical field. In phakic patients with clear lens, visualization is not affected by opacities, but vitreous removal is challenging because the convexity of the posterior curvature of the lens interferes in reaching the anterior part of the vitreous base, in fact, in these particular cases ambidexterity in bimanual maneuvers is required.

\section{The surgeon advice}

In the vitreoretinal surgery the procedure is never standardized. Many patients at the time of surgery have already underwent cataract extraction. The surgeon must be able to perform vitrectomy both in pseudophakic and in phakic eyes with some important differences in the procedure. If cataract is present at time of vitreoretinal surgery, it is useful to remove it before vitrectomy to improve visualization. In triple procedures it is advisable to implant the IOL leaving anterior chamber filled with ophthalmic viscoelastic device (OVD) after the complete vitreous removal to avoid visual disturbances due to IOL edges. In pseudophakic patients having posterior capsule opacities, is advisable to perform posterior capsulotomy after the vitreous base shaving to avoid IOL fogging. If posterior capsulotomy is already present, OVD coating of the posterior side of the IOL is useful. A drawback that can compromise the retina visualization, although less frequent, is the passage of air into the anterior chamber through zonular dialysis. In this case, an expedient is to fill the anterior chamber completely with air to improve visualization. Air removal from the anterior chamber would not solve the problem, 
because it would recur. In rhegmatogenous retinal detachment, it is recommended to mark any break with endocautery before BSS-air exchange, otherwise it could be arduous to recognize each rupture after the retina has been reattached to the pigment epithelium. In air medium, laser treatment is effective with a lower amount of energy compared with BSS- infusion, allowing useful and safe Video. endolaser treatment around retinal openings and peripherally at $360^{\circ}$. Horizontal meridians should be spared as much as possible to preserve long ciliary nerves. As to the bi-blade, the surgeon can experience a higher control of the field of visualization because of the small amplitude of the device vibrations.

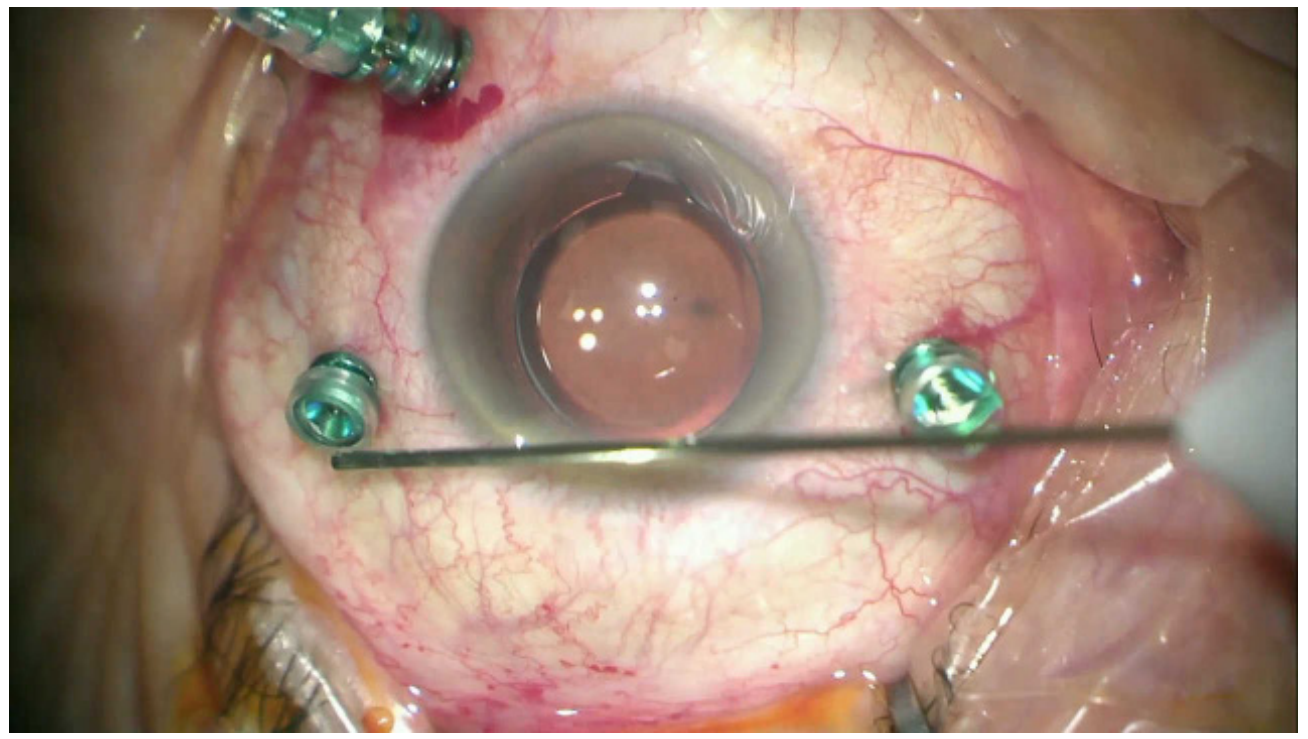

\section{Conclusion}

In conclusion, under air bi-blade assisted vitreous shaving is an effective combination that guarantees retinal stability and precise vitreous base shaving, pivotal phases for successful vitreoretinal surgery. It can be exploited especially for experienced surgeons in the management of challenging surgical cases.

\section{References}

1. Yuan J, Zhang LL, Lu YJ, Han MY, Yu AH, et al. (2017) Vitrectomy with internal limiting membrane peeling versus inverted internal limiting membrane flap technique for macular hole-induced retinal detachment: A systematic review of literature and meta-analysis. BMC Ophthalmology 17(1): 1-11.

2. Gui J, Ai L, Huang T (2020) Vitrectomy with or without internal limiting membrane peeling for myopic foveoschisis. BMC Ophthalmology 20(1): 1-18.

3. Michels RG, Rice TA, Rice EF (1983) Vitrectomy for diabetic vitreous hemorrhage. American journal of ophthalmology 95(1): 12-21.

4. van Overdam KA, van Etten PG, van Meurs JC, Manning SS (2019) Vitreous Wiping, a new technique for removal of vitreous cortex remnants during vitrectomy. Acta ophthalmologica 97(5): e747-e752.

5. González Saldivar G, Chow DR (2018) Update in Vitreoretinal Instrumentation. US Ophthalmic Review 11(2): 98.

6. Velez Montoya R, Guerrero Naranjo JL, Garcia Aguirre G, Morales Cantón V, Fromow Guerra J, et al. (2011) Perfluorocarbon-perfused 23 gauge three-dimensional vitrectomy for complicated diabetic tractional retinal detachment. Clinical ophthalmology (Auckland, NZ) 5: 1709-1715.

7. Sigler EJ, Randolph JC, Calzada JI (2013) Traction retinal detachment under silicone oil treated with two-port, 25-gauge pars plana vitrectomy and scissor segmentation in a child with familial exudative vitreoretinopathy. Retinal Cases and Brief Reports 7(3): 183-187.

8. Boral SK, Huang SS (2016) Interface vitrectomy. Retina \& Vitreous Surgery: A Practical Guide to Theory, Practice and Management. New Delhi: Jaypee Brothers Medical Publishers, pp. 180-183.
9. Altan T, Ozbilen KT, Cetin T, Kapran Z (2017) Results of peripheral vitrectomy under air in rhegmatogenous retinal detachment. Ophthalmic Surgery, Lasers and Imaging Retina. 48(1): 51-54.

10. Voleti VB, Gee CJ, Devin F, Hubschman JP (2012) Vitrectomy under air Retina 32(9): 1981-1982.

11. Romano MR, Stocchino A, Ferrara M, Lagazzo A, Repetto R (2018) Fluidics of single and double blade guillotine vitrectomy probes in balanced salt solution and artificial vitreous. Translational Vision Science and Technology 7(6): 19

12. Yau GL, Silva PS, Arrigg PG, Sun JK (2018) Postoperative complications of pars Plana vitrectomy for diabetic retinal disease. In Seminars in ophthalmology. Taylor \& Francis 33(1): 126-133.

13. Read SP, Fortun JA (2017) Visualization of the retina and vitreous during vitreoretinal surgery: new technologies. Current opinion in ophthalmology 28(3): 238-241.

14. Reibaldi M, Rizzo S, Avitabile T, Longo A, Toro, et al. (2014) Iatrogenic retinal breaks in 25-gauge vitrectomy under air compared with the standard 25-gauge system for macular diseases. Retina 34(8): 16171622.

15. Erdogan G, Unlu C, Karasu B, Kardes E, Ergin A (2016) Comparing peripheral vitrectomy under air and fluid infusion for primary rhegmatogenous retinal detachment. Retina 36(7): 1281-1284.

16. Karaçorlu M, Hocaoğlu M, Muslubaş IS, Ersöz MG, Arf S (2019) Outcomes of Vitrectomy Under Air for Idiopathic Macular Hole. Turk J Ophthalmol 49(6): 328-333.

17. (2019) Noticeable THE, Of A, Elite S, Iop L. Adaptive fluidics: a complete game changer. Inser TO Cataract Refract Surg TODAY Eur p. 1-2.

18. Porter RG, Peters JD, Bourke RD (2000) De-misting condensation on intraocular lenses. Ophthalmology 107(4): 778-782.

19. Hainswarth DP, Chen SN, Cox TA, Jaffe GJ (1996) Condensation on polymethylmethacrylate, acrylic polymer, and silicone intraocular lenses after fluid-air exchange in rabbits. Ophthalmology 103(9): 1410-1418. 